\title{
Cheap and Cheerful: Early Initiation of Oral Antihypertensives After ICH Saves Time and Money
}

\author{
Jennifer A. Frontera*
}

@ 2020 Springer Science+Business Media, LLC, part of Springer Nature and Neurocritical Care Society

The INTERACT2 and ATACH-II trials, both large, international, multi-center, randomized-controlled trials, evaluated the benefits of early aggressive blood pressure control following spontaneous intracerebral hemorrhage $(\mathrm{ICH})[1,2]$. While the INTERACT2 trial narrowly missed statistical significance on its primary outcome (modified Rankin [mRS] score of 3-6 at 90 days), a prespecified ordinal analysis of the mRS did demonstrate significantly better outcomes when blood pressure was aggressively lowered to a target systolic blood pressure $(\mathrm{SBP})<140 \mathrm{mmHg}$ and no differences in serious adverse events were identified [3]. The ATACH-II trial, conversely, failed to demonstrate a benefit of aggressive blood pressure control on the outcome of 3-month mRS 4-6. Although the trial aimed to compare a target $\mathrm{SBP}<180$ versus $<140 \mathrm{mmHg}$ in the first $24 \mathrm{~h}$ post-hemorrhage, the mean hourly minimum SBPs were much lower for each group $(120-125 \mathrm{mmHg}$ in the intensive group compared to SBP $140-145 \mathrm{mmHg}$ in the control group) [4]. There were no differences in treatmentrelated serious adverse events; however, renal adverse events of any severity were significantly higher in the treatment group. These results raised concerns that overly aggressive blood pressure lowering after $\mathrm{ICH}$ may lead to hypoperfusion injury in patients who may have right-shifted autoregulatory curves. Indeed, in one cohort study, $\mathrm{SBP}<120 \mathrm{mmHg}$ during the first $72 \mathrm{~h}$ post-ICH was associated with a significant risk of acute

\footnotetext{
*Correspondence: jennifer.frontera@nyulangone.org Department of Neurology, NYU Langone Health, 150 55th St., Brooklyn, NY 11231, USA
}

This comment refers to the article available at https://doi.org/10.1007/ s12028-020-00951-1. cerebral ischemia detected on magnetic resonance imaging, while no patient with a minimum $\mathrm{SBP} \geq 130 \mathrm{mmHg}$ had evidence of ischemia [5]. Furthermore, a substudy of INTERACT2 demonstrated a J-shaped curve wherein SBPs $<130$ or $>150 \mathrm{mmHg}$ during the first week after ICH were associated with worse functional outcomes [6]. To put the controversy over acute blood pressure lowering to rest, a pooled patient level prespecified meta-analysis of both the INTERACT 2 and ATACH-II trials $(N=3289)$ found that early blood pressure lowering was associated with significant improvements across a spectrum of functional outcome measures, reduced $\mathrm{ICH}$ expansion and was associated with fewer serious adverse events (all $P<0.0001$ ) [7]. Indeed, a linear dose response was identified such that every $10 \mathrm{mmHg}$ decrease in SBP improved the chances of functional recovery by $10 \%$. This study did, additionally, verify that blood pressure variability has a detrimental effect on outcome and should be avoided.

In the wake of these data, many practitioners have initiated blood pressure management protocols that typically involve the use of intravenous antihypertensives acutely following $\mathrm{ICH}$, which offer rapid onset, tight control and easy titration. However, the use of intravenous medications comes with a steep cost, including not only drug costs, but prolonged intensive care unit (ICU) length of stay (LOS) and nursing costs associated with monitoring. Downstream effects of prolonged ICU stays include unmeasurable costs associated with decreased throughput, lost admissions due to limited bed availability, ICUrelated complications (nosocomial infections, pressure ulcers) and reduced patient mobility. Since the median nationwide Medicare reimbursement for an intracranial hemorrhage hospital admission is only $\$ 13,000$ (based on diagnostic related grouping) $[8,9]$, cost containment is a critical goal for most hospital systems. 
In this issue, Zhu et al. present a practical, singlecenter, retrospective analysis of the impact of early administration of oral antihypertensives (within $24 \mathrm{~h}$ of hospital presentation) among patients with spontaneous, hypertensive ICH receiving intravenous nicardipine. The authors compared the early oral antihypertensive group $(N=90)$ to a control group $(N=76)$ in whom oral antihypertensives were initiated $>24 \mathrm{~h}$ after hospital presentation. They wisely excluded patients who underwent withdrawal of life-sustaining therapy or who only briefly received nicardipine. As expected, early initiation of oral antihypertensives led to shorter duration of nicardipine infusion with consequent reductions in drug cost by $50 \%$. What is impressive, however, is the significant drop in ICU LOS and decrease in cost of hospitalization. While these improvements may not be due solely to the initiation of early oral antihypertensives, the cost of nicardipine accounted for $60 \%$ of total hospital costs. Hence, reducing nicardipine use generated a lot of bang for your buck. As a bonus, there were no differences in adverse events, including renal events, between groups and hypotension occurred in only 1 patient (in the control group).

Strengths of this study, first and foremost, include the generalizable and practical ability for any ICU to institute similar early, aggressive oral blood pressure lowering protocols with demonstrated safety. Another strength is the robust handling of cost data, wherein nicardipine cost accounts for not only drug expense, but also acquisition, preparation, administration and monitoring costs. Similarly, hospitalization costs included both direct and indirect expenses. The multivariable analysis solidified the authors' conclusions by demonstrating both reductions in cost and ICU LOS.

Certain limitations of this study should be mentioned. First, the authors do not provide information on blood pressures achieved in each group (aside from the discharge blood pressures). Based on the aforementioned randomized trials, an imbalance in reaching target SBP or differing rates of blood pressure variability could significantly affect patient outcomes and neutralize any cost saving benefit. Second, as the authors acknowledge, the intervention and control groups were not well matched, suggesting an element of selection bias. Patients who were unable to initiate oral antihypertensives early had more severe neurological injury and thus, required feeding tubes, which delayed initiation of oral agents. The real question becomes whether patients with worse admission neurological status also had more refractory hypertension and hence higher nicardipine requirement, and higher hospitalization costs, which could occur irrespective of the timing of oral agent initiation. The authors did address this query in an earlier study of $48 \mathrm{ICH}$ patients with resistant hypertension, requiring $\geq 4$ blood pressure agents to maintain $\mathrm{SBP} \leq 140 \mathrm{mmHg}$ [10]. In this paper, there was no significant association of resistant hypertension with admission Glasgow Coma Score or ICH score. Furthermore, the authors did adjust for imbalances between groups in multivariable analysis and were able to demonstrate a significant effect on nicardipine use and cost. However, the magnitude of cost and LOS reduction is likely overestimated since the control group was significantly sicker than the intervention group and already prone to longer LOS and higher cost of hospitalization. Third, though the authors had originally intended to test the impact of a protocol they initiated in 2015 which mandated initiation of either amlodipine, lisinopril or both based on admission SBP [10], delays in dysphagia screening among over $12 \%$ of the patient population led them to include patients who received a heterogeneous potpourri of oral antihypertensives that were prescribed at the discretion of the treating physician. This heterogeneity injects a level of uncertainty in regards to the impact of drug choice upon outcome. Fourth, the authors don't tell us when intervention versus control patients was admitted to their ICU during the four-year study period. Major changes in LOS and cost along with improved functional outcomes could be due to changes in unit structure (e.g., transition from open to closed unit), introduction of a dedicated neurointensivist team, changes in clinical practice (e.g., minimally invasive clot evacuation) or other unmeasured variables.

Overall, despite these limitations, early initiation of oral antihypertensives in $\mathrm{ICH}$ patients appears to be both safe and cost effective, and should be a "no-brainer" for most neuro-intensivists.

\section{Source of Support}

None.

Conflict of interest

JA Frontera has nothing to disclose.

\section{Publisher's Note}

Springer Nature remains neutral with regard to jurisdictional claims in published maps and institutional affiliations.

Published online: 30 March 2020

\footnotetext{
References

1. Anderson CS, Heeley E, Huang Y, Wang J, Stapf C, Delcourt C, et al. Rapid blood-pressure lowering in patients with acute intracerebral hemorrhage. N Engl J Med. 2013:368(25):2355-65.

2. Qureshi Al, Palesch YY, Barsan WG, Hanley DF, Hsu CY, Martin RL, et al. Intensive blood-pressure lowering in patients with acute cerebral hemorrhage. N Engl J Med. 2016;375(11):1033-43.

3. Frontera JA. Blood pressure in intracerebral hemorrhage-how low should we go? N Engl J Med. 2013;368(25):2426-7.
} 
4. Frontera JA, Rabinstein AA. Intensive blood-pressure lowering in cerebral hemorrhage. N Engl J Med. 2016;375(23):e48.

5. Buletko AB, Thacker T, Cho SM, Mathew J, Thompson NR, Organek N, et al. Cerebral ischemia and deterioration with lower blood pressure target in intracerebral hemorrhage. Neurology. 2018;91(11):e1058-66.

6. Arima H, Heeley E, Delcourt C, Hirakawa Y, Wang X, Woodward M, et al. Optimal achieved blood pressure in acute intracerebral hemorrhage: INTERACT2. Neurology. 2015;84(5):464-71.

7. Moullaali TJ, Wang X, Martin RH, Shipes VB, Robinson TG, Chalmers J, et al. Blood pressure control and clinical outcomes in acute intracerebral haemorrhage: a preplanned pooled analysis of individual participant data. Lancet Neurol. 2019;18(9):857-64.
8. CMS.gov. Medicare-Provider-Charge-Data 2016. https://www.cms.gov/ Research-Statistics-Data-and-Systems/Statistics-Trends-and-Reports/ Medicare-Provider-Charge-Data/Inpatient2016.html.

9. Frontera JA, Bhatt P, Lalchan R, Yaghi S, Ahuja T, Papadopoulos J, et al. Cost comparison of andexanet versus prothrombin complex concentrates for direct factor $X a$ inhibitor reversal after hemorrhage. J Thromb Thrombolysis. 2020;49(1):121-31.

10. Hong D, Stradling D, Dastur CK, Akbari Y, Groysman L, Al-Khoury L, et al. Resistant hypertension after hypertensive intracerebral hemorrhage is associated with more medical interventions and longer hospital stays without affecting outcome. Front Neurol. 2017:8:184. 